\title{
Actitud de maestras y maestros hacia el trabajo cooperativo en el aprendizaje de la matemática
}

\author{
Luis Gerardo Meza Cascantel \\ Director, docente e investigador de la Escuela de Matemática del \\ Instituto Tecnológico de Costa Rica (ITCR) \\ Cartago, Costa Rica \\ Zuleyka Suárez Valdés-Ayala² \\ Docente e investigadora del Instituto Tecnológico de Costa Rica (ITCR) \\ Cartago, Costa Rica \\ Paulo García Delgado \\ Docente e investigador del Instituto Tecnológico de Costa Rica (ITCR) \\ Cartago, Costa Rica
}

Recibido 22 de febrero de 2010 • Aceptado 17 de marzo de 2010

Resumen. En este artículo se reportan los resultados más relevantes del proyecto de investigación "Actitud de maestras y maestros hacia el trabajo cooperativo en el aprendizaje de la matemática", desarrollado en la Escuela de Matemática del Instituto Tecnológico de Costa Rica, bajo el código 5402-14402201. Se mide, describe e interpreta la actitud de los maestros y las maestras hacia el trabajo cooperativo en el aprendizaje de la matemática, como resultado de la exposición a un taller en el que se empleó como estrategia metodológica el trabajo cooperativo para el aprendizaje de la matemática. La investigación contempló tanto técnicas cualitativas como cuantitativas.

Palabras clave. Actitud, aprendizaje cooperativo, matemática y actitud hacia el aprendizaje cooperativo de la matemática.

\footnotetext{
${ }^{1}$ Doctor en Educación con énfasis en Investigación Educativa (UNED), Costa Rica y Licenciado en la Enseñanza de la Matemática, Universidad Nacional (UNA), Costa Rica. Docente e investigador en la Escuela de Matemática del Instituto Tecnológico de Costa Rica (ITCR). También es docente en la División de Educología del Centro de Investigación y Docencia (CIDE), Universidad Nacional, Costa Rica. Actualmente, es Director de la Escuela de Matemática del Instituto Tecnológico de Costa Rica (ITCR). Correo electrónico: gemeza@itcr.ac.cr

${ }^{2}$ Máster en educación con énfasis en Docencia Universitaria de la Universidad Nacional (UNA), Costa Rica y Licenciada en la Enseñanza de la Matemática de la Universidad Nacional (UNA), Costa Rica. Laboró durante cuatro años en la enseñanza de la matemática en el nivel de primaria y durante siete años en la enseñanza de la matemática en el nivel de secundaria y educación diversificada. Actualmente, es docente e investigadora en la Escuela de Matemática del Instituto Tecnológico de Costa Rica (ITCR). Correo electrónico: zsuarez@itcr.ac.cr

${ }^{3}$ Licenciado en la Enseñanza de la Matemática de la Universidad Nacional (UNA), Costa Rica y egresado de la Maestría en Matemática Educativa de la Universidad de Costa Rica (UCR). Docente e investigador en la Escuela de Matemática del Instituto Tecnológico de Costa Rica (ITCR). Correo electrónico: pagarde@gmail.com
}

La Revista Electrónic@ Educare está indizada en el Catálogo LATINDEX. 
Abstract. This article reports the most important results of the research project "Attitudes of teachers to work cooperatively in the learning of mathematics", developed in the School of Mathematics at the Technological Institute of Costa Rica under the code 5402-1440-2201. There is a measurement, description and interpretation of the attitude of teachers to work cooperatively in the learning of mathematics, as a result of an exposure to a workshop in which a working cooperatively strategy for learning mathematics was used. The research involved both qualitative and quantitative techniques.

Key words. Attitude, cooperative learning, mathematics and attitude towards cooperative learning in mathematics.

\section{Introducción}

La enseñanza y el aprendizaje de la matemática han sido reconocidas como elementos sustantivos de los procesos educativos, y de importancia para el desarrollo científico y tecnológico de un país. En efecto, la Ley 7169 (Costa Rica. Asamblea legislativa. 1990) "Promoción del Desarrollo Científico y Tecnológico y Creación del MICYT (Ministerio de Ciencia y Tecnología)”, en el Artículo 3, inciso f, estipula, como objetivo específico para el desarrollo científico y tecnológico de Costa Rica, "Fomentar todas las actividades de apoyo al desarrollo científico y tecnológico sustantivo; los estudios de posgrado y la capacitación de recursos humanos, así como el mejoramiento de la enseñanza de las ciencias, las matemáticas y la educación técnica, lo mismo que la documentación e información científica y tecnológica" (p. 2)

El proyecto de investigación cuyos resultados se reseñan, se ubicó en este marco, y fue desarrollado con el fin de generar un conocimiento actualizado que permita fomentar otras actividades de investigación o extensión que se constituyan en un apoyo para el mejoramiento de la enseñanza y del aprendizaje de la matemática, en la educación primaria de Costa Rica.

La investigación se realizó en escuelas públicas del Cantón Central de Cartago, durante el primer semestre de 2009, con el propósito de estudiar la actitud de las maestras y los maestros hacia el aprendizaje cooperativo de la matemática como estrategia didáctica.

Como problema de investigación se formuló el siguiente: ¿Cuál es la actitud de los maestros y las maestras de la educación primaria hacia el aprendizaje cooperativo de la matemática de las y los estudiantes?

El objetivo general de la investigación fue "determinar la actitud de los maestros participantes hacia el aprendizaje cooperativo de la matemática", y se consideraron los siguientes objetivos específicos:

1. Desarrollar un taller de trabajo cooperativo para el aprendizaje de la matemática en ocho escuelas del Cantón Central de la provincia de Cartago, utilizando como material base el libro Háganlo juntos.

2. Describir e interpretar la actitud hacia el aprendizaje cooperativo de la matemática en los maestros y las maestras participantes, a partir de técnicas cualitativas.

3. Aplicar un diferencial semántico para medir la actitud hacia el trabajo cooperativo de los educadores y las educadoras participantes en los talleres desarrollados.

Los resultados obtenidos permiten conocer la actitud hacia el aprendizaje cooperativo de las maestras y los maestros, e identificar elementos de la cultura organizacional de las escuelas que pueden facilitar o dificultar el empleo del aprendizaje cooperativo, como estrategia didáctica en el aprendizaje de la matemática en la educación primaria. 


\section{Referentes teóricos}

\section{Sobre el constructo actitud}

En la investigación se adoptó como constructo teórico para el concepto de actitud, con fundamento en Gómez (2000), la disposición evaluativa (es decir, positiva o negativa), la cual determina las intenciones personales e influye en el comportamiento; ésta consta de tres componentes: a) una cognitiva que se manifiesta en las creencias subyacentes a dicha actitud, b) una componente afectiva que se manifiesta en los sentimientos de aceptación o de rechazo de la tarea o de la materia y c) una componente intencional o de tendencia a un cierto tipo de comportamiento.

Según esta misma autora, la definición anterior, que es de carácter genérico, puede ser complementada, cuando se trata de la matemática, mediante dos grandes categorías: actitudes hacia la matemática y actitudes matemáticas.

La primera categoría, o sea, las actitudes hacia la matemática (y que es la que nos interesaba en la investigación), se refiere a la valoración y al aprecio de esta disciplina y al interés por esta materia y por su aprendizaje, y se orientan más hacia la componente afectiva que hacia la cognitiva; la cual que se manifiesta en términos de interés, satisfacción, curiosidad, valoración, etc.

Las actitudes que comprenden este grupo, de acuerdo con Gómez (2000), pueden referirse a cualesquiera de los aspectos siguientes:

1. Actitud hacia la matemática y los matemáticos (aspectos sociales de la matemática).

2. Interés por el trabajo matemático y científico.

3. Actitud hacia las matemáticas como asignatura.

4. Actitud hacia determinadas partes de las matemáticas.

5. Actitud hacia los métodos de enseñanza.

\section{Sobre el trabajo cooperativo}

Compartimos con Yus (1997), la idea de que la cooperación es "la acción que se realiza juntamente con otro y otros individuos para conseguir un mismo fin" (s. p.). Este mismo autor, plantea que se da una estructura de trabajo cooperativo cuando, en un grupo, para alcanzar objetivos individuales, se requiere que el resto de él alcance los suyos.

Para que una persona sea cooperativa, debe desarrollar habilidades sociales y ser capaz de compartir con los demás. Lo anterior no significa que deba anularse la individualidad de cada persona, pues debemos estimular que ellos y ellas defiendan sus criterios.

Algunas ventajas del trabajo cooperativo, reseñadas por Yus (1997), son:

1. Mayor coordinación. Favorece la comunicación y la eficacia del trabajo.

2. Valoración positiva de los demás. Propicia un mayor entendimiento y ayuda mutua, así como la aceptación de otras ideas.

3. Mayor cohesión grupal. Se desarrolla más confianza y amistad entre los miembros del grupo.

4. Mayor maduración personal. Se desarrolla la capacidad de cooperar.

Es importante que al formar los grupos, el o la docente tenga en cuenta la heterogeneidad en cuanto a etnias, sexos y niveles de razonamiento, así como que estos grupos varíen para permitir un mayor contacto interpersonal. 
El profesor o la profesora debe intervenir en el desarrollo del trabajo grupal, sólo cuando sea estrictamente necesario; su función debe ser de facilitador o facilitadora, nunca de solucionador o solucionadora de la labor planteada.

Coincidimos con Yus (1997) en que, para llevar a cabo una discusión cooperativa, se deben tener en cuenta los siguientes aspectos:

1. Ser críticos con las ideas, no con las personas.

2. Centrarse en tomar la mejor decisión posible, no en ganar.

3. Animar a todos a participar.

4. Escuchar las ideas de todos, aunque no las comparta.

5. Aceptar nuevas ideas.

La cooperación no debe limitarse al ambiente educativo, sino que debe practicarse en los diferentes contextos en que nos desenvolvemos.

\section{La creación de ZDP (zonas de desarrollo próximo) en la interacción estudiantes/ estudiantes}

Señala Molina (1999), que en la corriente constructivista vigotskiana, el individuo posee dos niveles de desarrollo del conocimiento: la zona de desarrollo real o actual y la zona de desarrollo potencial o zona de desarrollo próximo. La primera consiste en todos los conocimientos que el individuo ha conseguido hasta ese momento, mientras que la segunda, en todos los conocimientos que el o la estudiante puede aprender y construir, con la ayuda de otras personas más expertas.

Según Vigotski (citado por Molina, 1999), la ZDP es la distancia entre el nivel real de desarrollo, determinado por la capacidad de resolver, independientemente, un problema y el nivel de desarrollo potencial, determinado por medio de la resolución de un problema con la guía de una persona más capacitada.

De acuerdo con Coll, Martín, Mauri, Miras, Onrubia, Solé et al. (1999), la interacción estudiantedocente es la principal fuente de creación de ZDP. No obstante, apunta este autor, el trabajo cooperativo entre estudiantes, bajo ciertas condiciones, también puede resultar importante en la creación de ZDP. Por lo anterior, cabe concluir que para potenciar la creación de ZDP, mediante la interacción entre las y los estudiantes, es preciso planificar, de manera muy cuidadosa y precisa, estas interacciones.

El aprendizaje cooperativo, permite una relación positiva entre las y los estudiantes. Esta relación positiva ocurre cuando trabajan juntos coordinan esfuerzos, obtienen mejores resultados y completan una tarea de manera más exitosa.

\section{Marco metodológico de la investigación}

\section{Tipo de investigación}

La investigación desarrollada es educativa mixta, pues en ella se combinó el enfoque cuantitativo con el cualitativo. Se ubica como una investigación de tipo descriptivo (según el enfoque cuantitativo), la cual combina técnicas de medición (propias del enfoque cuantitativo) con técnicas de recolección y el análisis de datos de tipo cualitativo, a saber: observación no participante y entrevistas en profundidad. 


\section{Sujetos y fuentes de información}

Los sujetos de la investigación fueron maestras y maestros de educación primaria de ocho escuelas públicas del Cantón Central de la provincia de Cartago.

La selección de los participantes se realizó mediante una técnica de muestreo no probabilístico denominada "muestreo por conveniencia" la cual, según McMillan y Schumacher (2005), consiste en seleccionar un conjunto de sujetos sobre la base de ser accesibles o adecuados. Esta modalidad de muestreo se justifica por cuanto la investigación requería de la disposición del centro educativo de participar en la investigación, y no sólo de los educadores en carácter individual.

\section{Acceso al campo}

De acuerdo con Rodríguez, Gil y García (1996), el acceso al campo significa la posibilidad de recoger un tipo de información que los participantes en un proceso educativo sólo proporcionan a aquellos en quienes confían, y que ocultan a los demás.

Para lograr el acceso al campo, que en esta investigación consistió en que la Escuela aceptara participar y que un número de sus maestros estuviera dispuesto a efectuar un taller de tres horas de duración, así como a completar un diferencial semántico y, eventualmente, participar en una entrevista en profundidad, se concertó una cita con la persona que dirige la Escuela.

En la reunión se expusieron los objetivos y los propósitos de la investigación, se explicó con detalle el proceso metodológico que se desarrollaría en la Escuela y se evacuaron las dudas que surgieron. Para ello, se presentó un documento escrito en el cual se expuso el propósito del estudio y otras consideraciones, para conocimiento del/la director/a.

Con el fin de tener claridad y garantía de lo negociado, se presentó al/la director/a un documento con carácter de propuesta, en el que se definieron las "reglas de juego" en cuanto al desarrollo de la investigación, y en el que, también, se expresó el tipo de apoyo que requeríamos de la institución y de los maestros participantes.

Sólo en una de las Escuelas hubo necesidad de renegociar las condiciones, dado que el Director propuso que se realizaran dos talleres debido a que la cantidad de maestros/as superaba los 50 .

Posteriormente, una vez logrado el permiso, en cada caso, se negoció la fecha y la hora en que se realizaría el taller y las otras actividades de la investigación.

\section{Instrumentos y técnicas para la recolección de los datos}

\section{Fase cuantitativa}

La recolección de los datos en la fase cuantitativa se realizó con la aplicación de un diferencial semántico, diseñado por los investigadores.

La validez del instrumento, entendida como el grado en el cual el instrumento mide lo que realmente debe medir, se estableció mediante el juicio de once expertos (jueces). El grupo de jueces estuvo integrado por profesionales en el campo de la enseñanza de la matemática y de otros campos del conocimiento, con experiencia en el diseño de instrumentos de medición.

A cada uno de los jueces se le entregó, además de una copia de la primera versión del diferencial semántico, un conjunto de instrucciones sobre lo que se esperaba de ellos, información de la investigación en desarrollo y una hoja de registro para que evaluaran el instrumento. 
A cada juez se le pidió que calificara cada una de las parejas de objetivos bipolares con 1, 2 o 3 , según las siguientes especificaciones:

\begin{tabular}{|l|l|}
\hline 1 & El par de adjetivos debe ser eliminado del instrumento \\
\hline 2 & El par de adjetivos puede ser mantenido en el instrumento pero se debe mejorar \\
\hline 3 & El par de adjetivos puede ser mantenido sin necesidad de modificación \\
\hline
\end{tabular}

Las recomendaciones de los jueces fueron valoradas mediante la aplicación de los siguientes criterios:

1. Las parejas que obtuvieron más del $70 \%$ de valores 3 , se mantuvieron en el instrumento sin modificaciones.

2. Aquellas parejas que obtuvieron más del $70 \%$ de valores 1 fueron eliminadas del instrumento sin más consideraciones.

3. En cualquier otro caso, se hizo una revisión de la pareja de adjetivos bipolares para evaluar la posibilidad de mejorarla, tomando en cuenta las recomendaciones de los jueces. En aquellos casos en que se consideró que no era posible mejorarla, se decidió eliminarla del instrumento.

Finalizado el proceso de validación, se eliminaron algunas parejas de adjetivos bipolares, y quedó la versión definitiva del instrumento, la cual está integrada por 11 parejas.

La confiabilidad del instrumento que, de acuerdo con Hernández, Fernández y Baptista (2006), se refiere al grado en que su aplicación repetida al mismo sujeto u objeto produce resultados iguales, fue establecida mediante la técnica del alfa de Cronbach.

El Alfa de Cronbach es un estadístico de amplio uso en investigaciones educativas, que produce un valor entre 0 y 1 . Un valor de 1 implica una confiabilidad perfecta y un valor de 0 , una confiabilidad nula.

Aunque no existe consenso sobre cuál es el valor mínimo de alfa que debe aceptarse para considerar que el instrumento es confiable, seguimos las recomendaciones de García (2006), quien propone una confiabilidad mínima de 0.90 para tomar decisiones sobre la vida de las personas, y una confiabilidad mínima de 0.50 para propósitos de investigación.

Mediante la aplicación de la técnica del Alfa de Cronbach y la utilización del programa SPSS, versión 15 , se obtuvo un valor de 0,762 , razón por la cual se consideró que el instrumento mostraba una confiabilidad aceptable.

\section{Fase cualitativa}

Con base en el planteamiento de Rodríguez et al. (1996), quienes sostienen que la investigación cualitativa puede realizarse no sólo preguntando a las personas implicadas en cualquier hecho o fenómeno social, sino también observando, utilizamos dos tipos de técnicas para la recolección de la información de tipo cualitativo: la observación no participante y la entrevista en profundidad.

Utilizamos la observación no participante porque, de acuerdo con estos autores, esta técnica permite obtener información sobre un fenómeno o acontecimiento tal y como éste se produce. Esta técnica, de acuerdo con Rodríguez et al. (1996), es uno de los procedimientos de observación más utilizados en la investigación cualitativa y uno de los elementos más característicos de este tipo de investigación. 
La observación no participante estuvo a cargo de los investigadores Meza y García, y la investigadora Suárez se encargó de guiar el desarrollo de los talleres en todos los casos.

Las figuras 1 y 2 muestran a varios docentes trabajando, de manera cooperativa, en dos talleres diferentes.

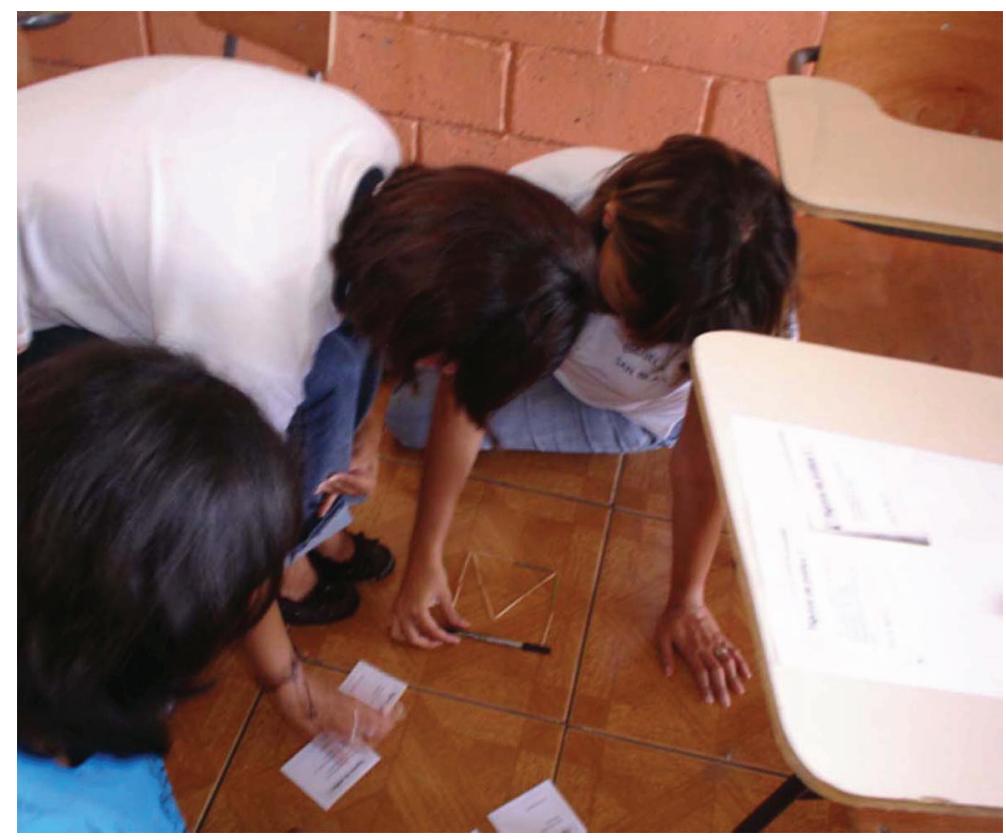

Figura 1. Docentes realizando un ejercicio de Geometría.

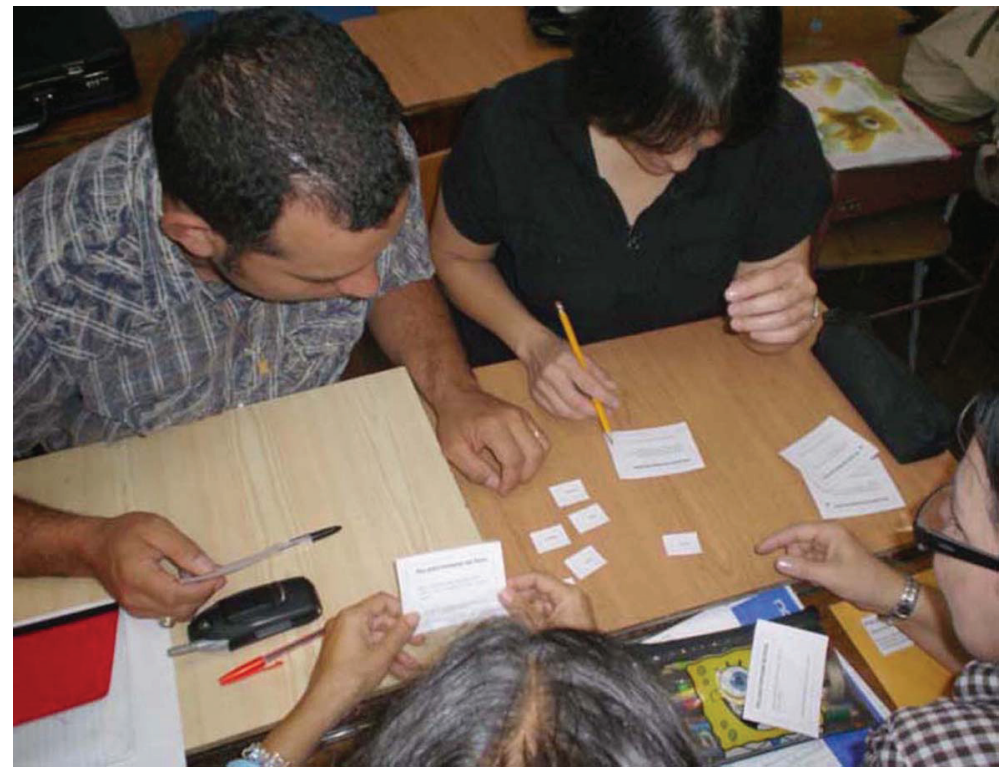

Figura 2. Docentes llevando a cabo un ejercicio de Lógica.

También utilizamos la entrevista en profundidad. De acuerdo con Rodríguez et al. (1996), en la entrevista en profundidad, el entrevistador desea obtener información sobre determinado problema y, a partir de él, establece una lista de temas, en relación con los que focaliza la entrevista. 
Entre los instrumentos utilizados para la recolección de la información en esta fase, se consideraron la cámara fotográfica (digital), con la cual se tomaron fotografías de algunos momentos del trabajo de las y los maestros en el desarrollo de los talleres, la grabadora de audio con la cual se grabaron todas las entrevistas en profundidad y cuadernos de apuntes (o libretas de notas), en los que se registraron las notas crudas tomadas en cada una de las observaciones realizadas.

\section{Análisis de los datos}

\section{Fase cuantitativa}

El análisis de los datos, en la fase cuantitativa, se realizó según los procedimientos ordinarios para el análisis de los datos obtenidos con diferenciales semánticos. Para ello, se utilizó el programa SPSS versión 15, con el fin de realizar un análisis de los estadísticos descriptores. Para facilitar el análisis y la interpretación de los datos, se utilizó la media como indicador de la medición de la actitud en cada una de las parejas de adjetivos bipolares. Con el fin de facilitar la interpretación de los valores de las medias, se procedió a definir las siguientes categorías, de acuerdo con el valor p de la media de cada pareja de adjetivos bipolares:

$1<\mathrm{p}<2$ : actitud muy negativa

$2<\mathrm{p}<3$ : actitud negativa moderada

$3<\mathrm{p}<4$ : actitud negativa baja

$4<\mathrm{p}<5$ : actitud positiva baja

$5<\mathrm{p}<6$ : actitud positiva moderada

$6<\mathrm{p}<7$ : actitud muy positiva

En la investigación se pudo captar la actitud de las maestras y de los maestros referentes al aprendizaje cooperativo de la matemática, de acuerdo con las categorías definidas para cada una de las parejas de adjetivos bipolares (véase Tabla 1).

Tabla 1

Opinión de las maestras y los maestros, sobre el aprendizaje cooperativo en la matemática

\begin{tabular}{|l|c|c|l|}
\hline \multicolumn{1}{|c|}{ Adjetivos bipolares } & Media & Desviación típica & \multicolumn{1}{c|}{ Valoración } \\
\hline INÚTIL-ÚTIL & 6,97 &, 173 & Muy positiva \\
\hline DIFÍCIL-FÁCIL & 5,35 & 1,534 & Positiva moderada \\
\hline ABURRIDO-DIVERTIDO & 6,71 &, 674 & Muy positiva \\
\hline FRUSTRANTE-MOTIVADOR & 6,52 &, 881 & Muy positiva \\
\hline ESTRESANTE-RELAJANTE & 5,64 & 1,464 & Positiva moderada \\
\hline COMPLICADO-SENCILLO & 5,21 & 1,463 & Positiva moderada \\
\hline INNECESARIO-NECESARIO & 6,80 &, 503 & Muy positiva \\
\hline DESAGRADABLE-AGRADABLE & 6,73 &, 621 & Muy positiva \\
\hline IRRELEVANTE-RELEVANTE & 6,58 &, 929 & Muy positiva \\
\hline INAPLICABLE-APLICABLE & 6,79 &, 569 & Muy positiva \\
\hline TRADICIONAL-INNOVADOR & 6,71 &, 655 & Muy positiva \\
\hline
\end{tabular}

Nota. Según el análisis de los datos obtenidos con diferenciales semánticos. 
De estos datos se desprende que las maestras y los maestros tienen una actitud muy positiva acerca del aprendizaje cooperativo en la matemática, pues consideran que es útil, divertido, motivador, necesario, agradable, relevante, aplicable e innovador. También, tienen una actitud positiva, aunque moderada, acerca de que el aprendizaje cooperativo de la matemática es fácil, relajante y sencillo.

Las maestras y los maestros no mostraron una actitud negativa en ninguna de las parejas de adjetivos.

\section{Fase cualitativa}

El análisis de los datos en una investigación cualitativa, de acuerdo con Rodríguez et al. (1996), no sigue un patrón lineal. Este proceso, indican, se realiza de manera simultánea con otras tareas, tales como la recogida de datos o la redacción del informe.

Como apuntan Taylor y Bogdan (1986), una buena regla por considerar en la toma de notas de campo es pensar que "si no está escrito, no sucedió nunca". Los profesores Meza y García fueron los encargados de redactar las bitácoras de los talleres y, posteriormente, entre los tres investigadores se hizo una puesta en común para consensuar criterios.

La categorización de los datos, de acuerdo con Rodríguez et al. (1996), constituye una importante herramienta en el análisis de los datos cualitativos y hace posible clasificar conceptualmente las unidades que son cubiertas por un mismo tópico. Para estos autores, una cuestión importante en esta etapa es establecer las categorías por emplear, e indican que éstas pueden estar predefinidas por el analista o, por el contrario, pueden surgir a medida que se analizan los datos.

Las categorías de análisis utilizadas fueron las siguientes:

1. Disposición de los maestros sobre la enseñanza y el aprendizaje de la matemática

2. Fines de la enseñanza de la matemática que asumen los maestros

3. Facilidades y obstáculos identificados por los maestros, para implantar el aprendizaje de la matemática mediante el trabajo cooperativo

Un/a maestro/a que manifieste una actitud negativa hacia la enseñanza y el aprendizaje de la matemática, no parece ser, al menos en principio, un/a buen/a candidato/a a mostrar una actitud positiva hacia el aprendizaje cooperativo de la matemática. Partiendo de esta premisa, en la investigación nos interesamos por develar elementos que nos permitieran construir una visión sobre la actitud de los/as maestros/as hacia la enseñanza y el aprendizaje de la matemática.

El testimonio de los/as maestros/as, obtenido de las entrevistas en profundidad, nos permitió acercarnos a la percepción que sobre la enseñanza y el aprendizaje de la matemática tenían los/as maestros/as protagonistas de la investigación.

Para conocer la disposición de los/as maestros/as sobre la enseñanza y el aprendizaje de la matemática, tenemos que darles voz: 


\section{Para Ileana}

"la Matemática es todo. Uno no puede vivir sin Matemática, si no saben cuáles son las tasas, cómo van a medir y todo lo que son distancias, todo lo que son los espacios, los conceptos espacio-temporales, conceptos pre numéricos. En Estudios Sociales cuando tienen que hacer las coordenadas geográficas tienen que tomar en cuenta Matemática; en Ciencias cuando tienen que hacer cosas tienen que tomar en cuenta la Matemática, aun en Español, cómo llamo las de dos sílabas, cómo se llaman las de tres sílabas".

\section{Para Grettel}

"cuando tomamos un programa de estudio, nos preguntamos: ¿qué tanto le va a servir para la vida lo que vamos a enseñar a nuestros estudiantes?, ojalá tengamos conciencia de ello y planifiquemos actividades que les permitan interiorizar adecuadamente los conocimientos que debe adquirir, tomando en cuenta los procesos mentales que necesita desarrollar para adquirir cierto conocimiento, es decir, ir de lo concreto a lo gráfico y, por último, a lo abstracto y lo pueda aplicar en situaciones cotidianas".

Yamileth, por su parte, agrega al fin práctico elementos del formativo cuando afirma que

"todo lo que nosotros aprendamos en el paso de los años es para la vida, para podernos defender, de una u otra manera, en cualquier situación a la que nos vayamos a enfrentar, y la Matemática con mucho más razón porque cuando se presenta un problema equis ya cuando somos adultos, uno tiene que tener tenacidad para poder enfrentarse a esas situaciones que no se pueden dejar botadas. Y como la Matemática se aplica en todo, tiene que haber tenacidad para aprenderla. Para poder aplicarla".

De las manifestaciones de los/as protagonistas, podemos intuir que las/os maestros/as tienen una concepción de la enseñanza y del aprendizaje de la matemática que enfatiza en los aspectos prácticos, mas, también, asoman cuestiones ligadas a la parte formativa. Estas dos finalidades que justifican la enseñanza y el aprendizaje de la matemática son claramente destacadas en la literatura especializada, como razones que justifican el aprendizaje de la matemática en la educación formal.

Esta reseña de algunos de los planteamientos de las/os maestras/os nos permite inferir una actitud positiva hacia la matemática; un reconocimiento a la importancia de la disciplina y a la necesidad de la matemática en la vida cotidiana. Consecuentemente, podemos deducir que las maestras y los maestros manifestaron una identificación con la matemática como disciplina útil, necesaria, de aplicación en la vida diaria, lo que nos permite interpretar su actitud hacia la enseñanza y el aprendizaje de esta disciplina como positiva.

Tal vez no sea de extrañar que el fin instrumental no esté destacado por las/os maestras/os, a causa de que este fin lo que promueve es la enseñanza y el aprendizaje de la matemática como lenguaje de otros campos del saber. Aunque, ciertamente, esto también puede darse en la educación primaria, al parecer las/os maestras/os no perciben en los contenidos abordados en ese nivel educativo ese carácter de lenguaje de las otras disciplinas.

Una propuesta como la que hicimos a las/os maestras/os de utilizar la enseñanza y el aprendizaje cooperativo de la matemática, especialmente porque la propuesta hace uso de un material didáctico 
particular, debe considerar los elementos de la cultura organizacional de la Escuela en la que se desarrolla.

De acuerdo con Robbins (1987), se entiende por cultura organizacional el sistema de significados común entre las y los integrantes que distingue a una organización de otras. La cultura organizacional viene a estar constituida por una serie de fenómenos invisibles que afectan el comportamiento de una organización.

Tal como lo ha evidenciado Meza (2003), la cultura organizacional de una institución educativa ofrece oportunidades y también obstáculos, para el desarrollo de procesos de innovación educativa ligados a la enseñanza y al aprendizaje de la matemática.

Por todo ello, en esta investigación nos interesamos por develar algunos aspectos de la cultura organizacional de las Escuelas, y, eventualmente, otros elementos de la institución, que tienen potencial para facilitar la implantación del aprendizaje cooperativo de la matemática o para dificultarlo.

Uno de los elementos que podría interferir con la innovación educativa que supone la adopción del aprendizaje cooperativo de la matemática, es la falta de trabajo coordinado entre las/los maestras/os.

\section{Minoska indica que}

“trabajamos en forma individual. En el caso mío yo imparto Matemáticas a dos grupos, con mi otra compañera no comparto ni técnicas ni lo que hago. Igual yo no me doy cuenta por dónde va ella en su campo, ni ella se da cuenta por dónde yo voy. Nos guiamos por el programa de estudios del Ministerio de Educación”.

Varias docentes opinaron de la misma forma que ella.

La condición del trabajo, predominantemente, individual, la valoramos como un elemento que podría afectar, de forma negativa, la innovación educativa relacionada con el aprendizaje cooperativo de la matemática. Tal como se ha evidenciado en investigaciones desarrolladas por Meza (2003), en los procesos de innovación educativa se hace necesario el acompañamiento de las y los docentes innovadores. El trabajo coordinado entre los propios educadores es una de las estrategias recomendadas para lograrlo.

Otro elemento que podría dificultar una innovación educativa como la propuesta, tiene que ver con la falta de tiempo que siente el/la maestro/a que le aqueja para cumplir con el programa de estudios. El testimonio de Yamileth es claro, en este sentido

"Nosotros estamos sujetos a un programa, no nos detenemos a hacer actividades tan dinámicas y de trabajo en equipo, porque tenemos que cumplir con un programa".

Afortunadamente, posiciones como la que expresa Grettel nos muestra que los/as propios/ as maestros/as pueden encontrar una salida ante la sensación de falta de tiempo. En efecto, dice Grettel:

"Pienso que el tiempo y otras variables como la cantidad de contenidos de los programas de estudio, las pruebas escritas o sumativas y demás actividades curriculares hacen que los maestros sintamos estrés. Pero si el maestro cuenta con actividades debidamente planificadas, acordes con los contenidos del programa y en el nivel que impartimos, se puede destinar una lección semanal (40 minutos) para ejecutarlas, porque no sólo contribuyen al desarrollo de 
habilidades y destrezas matemáticas, sino que fortalecen valores importantísimos como la convivencia, la cooperación, la tolerancia, el respeto y otros que permiten trabajar en forma cooperativa".

Por su parte, Minoska nos ayuda a descubrir otro elemento que podría afectar de manera negativa el empleo del trabajo cooperativo en la enseñanza y el aprendizaje de la matemática. Ella apunta que

“... otro obstáculo que yo creo que puede darse es que a los alumnos no les gusta trabajar, entonces pienso que ahí si se vería afectado el trabajo cooperativo, cuando algunos, por determinadas circunstancias, no quieren hacer nada y se atienen a que los demás les resuelvan los problemas".

Un elemento inesperado que surgió durante el desarrollo de los talleres fue el ruido generado en el aula. Efectivamente, en los talleres realizados con muchos participantes, por lo general, con número cercano a 20 personas, notamos que el desarrollo de las actividades propuestas generaba un ruido apreciable. Éste se genera porque las personas tienen que hablar para negociar, coordinar, aportar, diferir y resolver. Consecuentemente, le dedicamos atención a conocer cómo perciben las/ os maestras/os este elemento.

Sobre este tema Ileana dice:

"a mí, el ruido me desconcentra terriblemente. Definitivamente la organización dentro del aula es fundamental, el establecimiento de normas, el establecimiento del respeto, del espacio verbal y físico de los demás grupos al estar trabajando es muy importante, porque si no, de verdad, afecta montones".

Yamileth tiene una visión más positiva. Ella lo plantea de la siguiente manera:

"yo creo que está en uno que los chiquitos no se salgan de la norma; yo creo que mientras estén trabajando no veo que se forme tanto ruido, yo no le veo tanto problema a eso".

De lo expuesto por las/os maestras/os, y de las observaciones realizadas durante el desarrollo de los talleres, consideramos que el factor ruido surge como un factor que podría generar conflicto en el desarrollo de las actividades de enseñanza y de aprendizaje de la matemática de forma cooperativa. No obstante, de estas manifestaciones también se desprende que es un factor que puede ser minimizado en sus efectos negativos, si se adoptan medidas de manera oportuna.

Como parte de la investigación nos interesamos por detectar elementos que las/os maestras/os pudieran señalar como necesarios para poder utilizar el aprendizaje cooperativo, según la modalidad didáctica particular que les propusimos. Uno de ellos, que afloró muy rápidamente, tiene que ver con la capacitación que las/os maestras/os solicitan.

Grettel lo expone de la siguiente manera:

"Tal vez es necesario una preparación o capacitación a los docentes para conocer estas alternativas y encontrar la relación con los contenidos de los programas de estudio". 
Planteamiento que refuerza al decir: "es importante que nos capaciten, porque, muchas veces, no encontramos actividades fáciles e innovadoras que permitan reforzar los aprendizajes adquiridos por los estudiantes".

Para nosotros, el acompañamiento al docente que pretende innovar es necesario porque, como indica Feldman (1999), "el profesor no se "hace" conservador en el trabajo, sino que "vuelve" a ser conservador; pues ante la incertidumbre en la que se desarrolla su práctica, recurre a actitudes y a principios de acción aprendidos de sus maestros durante su formación.

Las demandas de trabajo adicional que podría enfrentar el/la maestro/a al aplicar una metodología como la propuesta, no podía ser dejada de lado en la investigación. Esto por cuanto teníamos como propósito acercarnos a la realidad de la Escuela, mirarla sin prejuicios y sin acomodos, tratando de develar tanto los elementos que, positivamente, podrían apoyar la innovación educativa que le proponíamos a las/os maestras/os, como aquellos que, por el contrario, podrían dificultarla.

\section{Para Minoska:}

"Lo que sea para innovar o lo que haga un aula más creativa, si uno ve que los chiquillos están contentos, que están trabajando, yo no lo vería como una obligación, sino que es parte de la lección que se está dando en forma diferente, con una metodología diferente y que, igual, da sus frutos. Obviamente, va a demandar algo más, pero es parte de las labores que tiene uno, porque si yo voy a dar una lección, pues igual tengo que planificarla."

Esta disposición positiva que muestran las/os maestras/os no debe ocultarnos que los procesos de innovación educativa conllevan, al menos potencialmente, más obligaciones para los/as educadores/as. Una actitud positiva como la que muestran las/os maestras/os aparece como elemento que aminora el efecto negativo que esa situación genera.

En el caso particular de la estrategia de enseñanza y de aprendizaje de la matemática mediante trabajo cooperativo, es posible atenuar el trabajo adicional que acarrea utilizando materiales preparados por universidades o por otras instituciones, como es el caso del libro titulado Háganlo juntos. De esta manera, el/la maestro/a encuentra materiales preparados y validados que le permiten implantar la estrategia, sin tener que dedicar mucho tiempo en la planificación.

Por la actualidad del tema, no quedaron sin explorar las repercusiones que el empleo de una estrategia didáctica como la propuesta, tiene sobre las adecuaciones curriculares.

Para Izel,

“... lo más importante sería que el niño se integre a la actividad que está haciendo el resto de sus compañeros, la actividad más simple será para él, pero igual irá a trabajar en grupo, igual irá a pensar que el resto de sus compañeros, verdad, y que todos lo apoyen y que todos lo ayuden y él, así, ayudar a los demás".

Ángela, por su parte, se muestra muy optimista. Ella plantea al respecto:

“...no veo mejor posibilidad de incluir a un niño con necesidades educativas especiales que en un trabajo cooperativo, precisamente, porque es la mejor oportunidad que tenemos de que él desempeñe un rol positivo, un rol de provecho, como dice Milagro, un rol con el que él pueda 
aportar y en el que haya precisamente apoyo de sus compañeros para lograr un objetivo común; entonces, no veo mejor posibilidades de lograr esa inclusión que en un trabajo cooperativo".

Este tema de la adecuación curricular es muy complejo, y la investigación no permitió abordarlo con profundidad. No obstante, queda claro, por una parte, que la atención de los/as niños/as con necesidades educativas especiales es un asunto por considerar en la implantación del aprendizaje cooperativo de la matemática, para lo cual aún no tenemos respuestas concretas. Por otra, la motivación que mostraron las/os maestras/os con la opción metodológica propuesta, y la experiencia que han generado en la atención de los/as niños/as con necesidades educativas especiales, parece ofrecer un panorama, suficientemente, positivo sobre el particular.

Resulta de interés diferenciar, a partir de los planteamientos de las/os maestras/os la actitud hacia el trabajo cooperativo de la actitud sobre el taller realizado. Aunque, ciertamente, tienen relación, se trata de cuestiones distintas.

Yolanda opina, sobre el taller en que participó, lo siguiente:

"Me gustó bastante, en el caso de los niños que ellos comiencen a desarrollarse y a trabajar en grupo, a tener esa armonía en grupo y poder trabajar en conjunto, porque ahora cuesta mucho que ellos trabajen juntos, hay muchos individualistas, y se ha perdido mucho el trabajar en grupo".

Grettel agrega:

“...considero que la idea del taller era trabajar en forma cooperativa, los fines de esta actividad se cumplieron. Se puso en evidencia, que dos cabezas piensan mejor que una. Al trabajar en pequeños grupos, logramos que cada uno de los integrantes aportara sus ideas y sus conocimientos para la resolución de las actividades propuestas".

De acuerdo con los planteamientos de las/os maestras/os podemos inferir una actitud positiva, tanto hacia el trabajo cooperativo como estrategia didáctica, como hacia el taller que vivenciaron.

\section{Discusión de resultados y conclusiones}

La investigación realizada permitió acercarnos a la realidad de la escuela costarricense, y constatar, gracias al contacto directo establecido con las/os maestras/os, que ellas/os piensan que el aprendizaje cooperativo de la matemática es útil, divertido, motivador, necesario, agradable, relevante, aplicable, innovador. Pudimos constatar, también, que tienen una actitud positiva, aunque moderada, acerca de que el aprendizaje cooperativo de la matemática es fácil, relajante y sencillo.

Además, la investigación nos permite inferir que las/os maestras/os tienen una actitud positiva hacia la matemática como disciplina, y reconocen que ésta es importante, en general, y en la vida cotidiana, en particular.

Desde un punto de vista más pedagógico, podemos intuir que las/os maestras/os tienen una concepción de la enseñanza y del aprendizaje de la matemática centrado en los aspectos prácticos, esto es, en la necesidad de conocer matemática porque se requiere de ciertas destrezas y de conocimientos concretos para poder enfrentar problemas del mundo laboral, en particular, y de la vida ordinaria, en general. Mas, también, asoman cuestiones ligadas a la parte formativa, lo que constituye 
un aliciente importante, porque el fin formativo resulta ser, según un cierto consenso especializado, el valorado como más importante.

Asimismo, la investigación nos muestra que la cultura organizacional de cada escuela puede ofrecer elementos potencialmente favorecedores, que hagan del aprendizaje cooperativo de la matemática una estrategia didáctica exitosa. Pero, también, muestra que pueden existir elementos en esa cultura organizacional que afecten negativamente la eficacia de una estrategia didáctica como la propuesta.

Al respecto de esto último, tenemos que la predominancia del trabajo individual de las/os maestras/os, puede constituir una amenaza al proceso de innovación ligado al empleo del aprendizaje cooperativo de la matemática, por cuanto el acompañamiento que podría requerir un/a maestro/a innovador/a, de sus compañeros, podría no concretarse.

Dentro de ese mismo tipo de elementos, encontramos que la sensación que tienen las/os maestras/os de que el tiempo disponible no es suficiente para desarrollar el programa, así como la convicción de que el programa debe ser cumplido, es otra amenaza al desarrollo de actividades innovadoras. No obstante, es gratificante constatar que las/os maestras/os pueden generar espacios en su actividad para innovar, a pesar de la sensación descrita.

Desde una perspectiva más puntual, encontramos que el ruido que se genera en el aula donde se desarrollan procesos de enseñanza y de aprendizaje cooperativo de la matemática, puede constituir un obstáculo para el empleo de esa estrategia didáctica, especialmente, por las molestias que puede generar a otras/os maestras/os y a los/as alumnos/as que se encuentran cerca del aula en donde se genera el ruido. Considerando la dinámica propia de las escuelas, y los diseños ordinarios de los edificios de las instituciones educativas costarricenses, caracterizadas por la cercanía entre las aulas y otros recintos educativos, tenemos que el ruido que se genera en la aplicación de la estrategia propuesta, puede constituir un obstáculo para su efectiva implantación. Por ello, y tal como sucede en otros esfuerzos de innovación en educación, tenemos que ir más allá de la visión puramente didáctica de la propuesta, y atrevernos a mirar los procesos de enseñanza y de aprendizaje de la matemática en otros ámbitos físicos no tradicionales.

La investigación también devela que las/os maestras/os que utilicen una estrategia de aprendizaje cooperativo de la matemática, podrían experimentar una mayor carga de trabajo. No obstante, con el empleo de materiales, expresamente, preparados del tipo Háganlo juntos, podrían reducir el trabajo adicional. En este punto, nace un desafío para los educadores costarricenses. Tenemos que ser capaces de generar materiales similares a la obra Háganlo juntos, nacidos de nuestra realidad, alimentados con nuestra creatividad y nutrido de nuestras vivencias. Éste es un desafío que debemos asumir tanto los investigadores como los docentes.

Finalmente, la investigación también nos permite tomar conciencia de dos elementos adicionales. Todos ellos, a nuestro juicio, relevantes. En primer lugar, tenemos que tomar en cuenta que en la implantación de la estrategia de aprendizaje cooperativo de la matemática, el/la educador/a debe considerar que no todos/as los/as estudiantes están deseosos/as de participar en procesos de innovación educativa. Asumir que la innovación, por el simple hecho de serlo, encantará a todos/as los/ as estudiantes puede ser una actitud ingenua. La experiencia con otros procesos de innovación nos enseña que no todos/as los/as estudiantes, y no todo el tiempo, estarán encantados de participar en ese tipo de procesos.

En segundo lugar, la necesidad de capacitación, y de acompañamiento durante el proceso de innovación, parecen ser elementos críticos para que las/os maestras/os incursionen, con decisión, en el empleo de la estrategia didáctica del trabajo cooperativo en la enseñanza y el aprendizaje de la matemática. Los resultados de otras investigaciones concuerdan con los nuestros: la innovación en educación requiere de procesos de capacitación y de acompañamiento. 
Como elemento integrador de lo dicho, podemos inferir una actitud positiva de los/as maestros/ as, tanto hacia el trabajo cooperativo como estrategia didáctica, como hacia el taller que se desarrolló.

Lo planteado anteriormente, se concreta, entonces, en las siguientes conclusiones:

1. Las/os maestras/os piensan que el aprendizaje cooperativo de la matemática es útil, divertido, motivador, necesario, agradable, relevante, aplicable e innovador.

2. Las/os maestras/os tienen una actitud positiva hacia la matemática; un reconocimiento a la importancia de la disciplina y a la necesidad de la matemática en la vida cotidiana.

3. Las/os maestras/os tienen una concepción de la enseñanza y del aprendizaje de la matemática que enfatiza en los aspectos prácticos, pero también asoman cuestiones ligadas a la parte formativa.

4. La cultura organizacional de cada escuela parece ofrecer elementos que pueden incidir en el empleo del aprendizaje cooperativo de la matemática como estrategia didáctica.

5. La predominancia del trabajo individual de las/os maestras/os puede constituir una amenaza al proceso de innovación ligado al empleo del aprendizaje cooperativo de la matemática, por cuanto el acompañamiento que podría requerir un/a maestro/a innovador/a de sus compañeros/as podría no concretarse.

6. La sensación que tienen las/os maestras/os de que el tiempo disponible no es suficiente para desarrollar el programa, así como la convicción de que el programa debe ser cumplido, es otra amenaza al desarrollo de actividades innovadoras. No obstante, también, tenemos el hallazgo de que las/os maestras/os pueden generar espacios en su actividad para innovar, a pesar de la sensación descrita.

7. El ruido que se genera en el aula en la que se desarrollan procesos de enseñanza y de aprendizaje cooperativo de la matemática, puede constituir un obstáculo para el empleo de esa estrategia didáctica, especialmente, por las molestias que puede generar a otras/os maestras/os y a los/as alumnos/as que se encuentran cerca del aula donde se genera el ruido.

8. Las/os maestras/os que utilicen una estrategia de aprendizaje cooperativo de la matemática, podrían experimentar una mayor carga de trabajo. No obstante, con el empleo de materiales expresamente preparados del tipo Háganlo juntos, podrían reducir el trabajo adicional.

9. En la implantación de la estrategia de aprendizaje cooperativo de la matemática, el/la educador/a debe considerar que no todos/as los/as estudiantes están deseosos/as de participar en procesos de innovación educativa.

10.La necesidad de capacitación, y de acompañamiento durante el proceso de innovación, parecen ser elementos críticos para que las/os maestras/os incursionen, con decisión, en el empleo de la estrategia didáctica del trabajo cooperativo en la enseñanza y el aprendizaje de la matemática.

\section{Referencias bibliográfícas}

Coll, C., Martín, E., Mauri, T., Miras, M., Onrubia, J., Solé, I., et al. (1999). El constructivismo en el aula. Barcelona, España: Graó.

Costa Rica. Asamblea Legislativa. (1990). Ley 7169. Promoción del Desarrollo Científico y Tecnológico y Creación del MICYT (Ministerio de Ciencia y Tecnología). Aprobada el 26 de junio de 1990. San José, Costa Rica: Autor. 
Feldman, D. (1999). Ayudar a enseñar. Buenos Aires, Argentina: AIQUE Grupo Editor.

García, C. (2006). La medición en ciencias sociales y en la psicología. En M. González \& R. Landeros (Comp.). Estadística con SPSS y metodología de la investigación. México: Editorial Trillas.

Gómez, I. (2000). Matemática emocional. Los afectos en el aprendizaje matemático. Madrid, España: Narcea.

Hernández, R., Fernández, C. \& Baptista, P. (2006). Metodología de la investigación. México: McGraw Hill.

McMillan, J. \& Schumacher, S. (2005). Investigación educativa. (5 ed.). Madrid, España: Pearson Educación.

Meza, G. (2003). Hacia perfiles de cambio en la enseñanza y el aprendizaje de la matemática: un caso de estudio en sétimo año de un colegio oficial urbano. En: Revista Virtual de Posgrado de la UNED. Recuperado el 14 de octubre de 2008, de http://www.uned.ac.cr/sep/recursos/ investigaciones/ded.htm

Molina, Z. (1999, II semestre). Planificación, diseño y desarrollo curricular. Revista Umbral, 10, 4-27.

Robbins, S. (1987). Comportamiento organizacional. México: Prentice Hall.

Rodríguez, G., Gil, J. \& García, E. (1996). Metodología de la investigación cualitativa. Málaga, España: Ediciones ALJIBE.

Taylor, S. \& Bogdan, R. (1986). Introducción a los métodos cualitativos de investigación. Buenos Aires, Argentina: Paidós.

Yus, R. (1997). Desde la cooperación en la escuela a la cooperación para el desarrollo (Una relectura del pensamiento de Freinet en los albores del siglo XM). Educación, Desarrollo y participación democrática. Proyecto y tú... ¿Cómo lo ves? Recuperado el $1^{\circ}$ de setiembre de 2008, de http://www.fongdcam.org/manuales/educaciondesarrollo/datos/docs/A_docs/b_1_9 ryus.pdf 